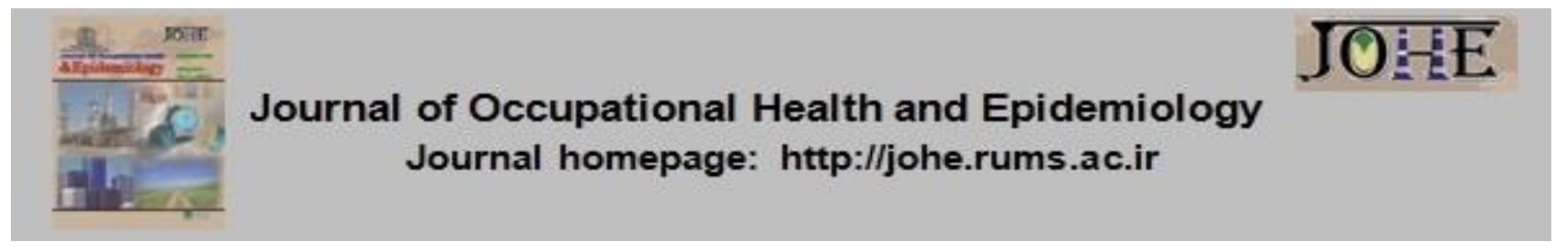

\title{
Comparing Rumination and Depression Scores in Employed and Unemployed Mothers of Children with Smartphone Addiction in Tehran, 2019-2020
}

\author{
Melina Jafarzadeh ${ }^{1}$, Mahnaz Cheshmi², Maryam Ranjbar Kolagari ${ }^{3}$, Roya Madadi ${ }^{4}$, Javad Seyed \\ Jafari $5^{*}$ \\ 1. MSc in Family Counseling, Ardakan University, Ardakan, Iran. \\ 2. MSc in Clinical Psychology, Family Therapy, Tehran University of Science and Culture, Tehran, Iran. \\ 3. MSc in Educational Psychology, Islamic Azad University, Sari Branch, Sari, Iran. \\ 4. MSc in Clinical Psychology, Master of Counseling and Guidance, Islamic Azad University, Roudehen Branch, Roudehen, Iran. \\ 5. PhD Candidate in Psychology, Allameh Tabataba'i University, Tehran, Iran.
}

\section{Article Info}

* Corresponding author:

Javad Sayed Jafari,

E-mail:

Javad-jafari90@yahoo.com

\section{Article history}

Received: Nov 2020

Accepted: Jan 2021

10.29252/johe.9.4.206

Print ISSN: 2251-8096 Online ISSN: 2252-0902

Peer review under responsibility of Journal of Occupational Health and Epidemiology
Citation: Jafarzadeh M, Cheshmi M, Ranjbar Kolagari M, Madadi R, Seyed Jafari J. Comparing Rumination and Depression Scores in Employed and Unemployed Mothers of Children with Smartphone Addiction in Tehran, 2019-2020. JOHE 2020; 9(4):206-12.

\begin{abstract}
Background: Smartphone addiction is a serious growing problem with similarities to game addiction and even drug addiction. Nowadays, a large number of Iranian children and teenagers find themselves addicted to their smartphones. This type of addiction has a serious impact on mental health among mothers. This study aimed to compare depression and rumination scores among employed and unemployed mothers of preschool children with smartphone addiction.

Materials and Methods: This is a descriptive correlational study, in which 150 mothers of preschool children were selected as the sample group. In this study, the Cellphone Overuse Scale (COS), the Beck Depression Questionnaire, and the Intellectual Rumination Questionnaire were utilized. In addition, the Pearson's correlation coefficient and the independent samples t-test methods were used for data analysis.

Results: According to the results, the mean and standard deviation of depression and rumination among employed and unemployed mothers were $(M=3.83, S D=3.78)$ and $(M=8.48, S D=7.83)$, respectively. In addition, the mean of rumination among unemployed mothers $(M=24.08 S D=13.65)$ was significantly higher than that among employed mothers $(M=14.42, S D=8.34)(p<0.05)$.

Conclusion: The findings provide the preliminary foundation for further studies on designing effective prevention programs to prevent depression and rumination among mothers with children addicted to smartphones.
\end{abstract}

Keywords: Rumination, Depression, Mothers, Unemployment, Preschool Children, Smartphone, Smartphone Addiction.

\section{Introduction}

Throughout history, many instances of advances have been successful in helping the human community, yet their negative effects have become evident over time [1]. Cellphones or handsets are efficient communication devices that Motorola first developed in 1973 and made them commercially available in 1984 [2]. Nowadays, addiction does not only apply to alcohol or substance abuse, yet addiction to gambling, the internet, sports, or even smartphones has become prevalent as well. These types of addiction fall under the category of behavioral addiction [3]. In 2014, 1.85 billion people used smartphones worldwide, which is expected to rise to 2.32 billion in 2017 and 2.87 billion in 2020 [4]. Smartphones provide numerous benefits, such as sociability, entertainment, information, time management, coping strategies, 
and maintenance of the social identity [5]. Besides, smartphones have become an important part of everyday life, with research showing that some people become so attached to their smartphones as to feel anxious when separated from them [6]. This device can even be soothing at times of stress by exerting a "safety blanket" effect, by which initial negative reactions to a stressor are diminished even in children who use it as an object of comfort, like a blanket [7].

Although smartphones provide convenience for our lives, we need to be aware of the negative consequences of their use, with smartphone addiction being one of the most worrisome factors. Smartphone addiction is a condition in which the use of smartphones becomes uncontrollable. Individuals with this type of addiction face social, psychological, and health challenges [8]. Research indicates that smartphone addiction is associated with various internalizing and behavioral problems. While a significant number of the findings point to negative outcomes of smartphones, there is a dearth of evidence concerning determinants of smartphone addiction. It is evident that teenagers have an intimate relationship with technology [9]. Because of the convenience provided by smartphones, humans have become more dependent on them [3]. Smartphone addiction harms children's social engagement. When children become addicted to smartphones, they do not participate in social activities. The negative effects indicate social implications of media addiction beyond psychological and practical implications [10]. Moreover, research shows that smartphone addiction has negative impacts on individuals' psychological and physical health as well as their academic and professional performance $[10,11]$. To examine the factors leading to smartphone addiction, past research focused on psychological factors and revealed that stress, shyness, loneliness, anxiety, and depression are positively correlated with smartphone addiction [10].

Researchers have conducted studies on the relationship between addiction and adolescent dependence on smartphones [6-11]. Nevertheless, no research has examined the impact of smartphone addiction on employed and nonemployed mothers. According to field research conducted by the present researchers, addiction to smartphones among children leads to bullying acts, isolation, lack of social interaction with peers, lack of interest in social games, depression, and anxiety [12]. These consequences, in turn, lead to psychological problems, such as depression and rumination, in their mothers.
Rumination, being a cognitive process characterized by repetitive thinking based on negative feelings and thoughts, is an effective indicator of the onset of internalizing symptoms, which has been found to occur in families [13]. Rumination, described as a cognitive process characterized by an abstract and repetitive way of thinking based on negative feelings and thoughts and their consequences, has recently emerged as a leading predictor of the onset of internalized disorders [14]. In fact, ruminants have rarely been investigated because of the powerful stressors causing negative thoughts and influencing them [13]. Michl et al reported in their longitudinal study that the level of rumination increased following stressful events [15]. In their study, smartphone addiction among children was considered as a stressor. Additionally, failure to find a solution for decreasing the use of smartphones by children increases the feeling of helplessness, thereby creating a vicious cycle that aggravates rumination [16]. Rumination exerts many effects on depression, thereby reducing optimism for the future [17]. In addition, rumination serves as a mechanism connecting stressful life experiences to symptoms of depression and anxiety [15]. Furthermore, effects of rumination on social problem solving worsen depression [18,19]. Accordingly, this study aims to compare depression and rumination among mothers of preschool children with smartphone addiction in Tehran in the period from 2019 to 2020.

\section{Materials and Methods}

The present study used a descriptive correlational design. The statistical population of this study included all employed and unemployed mothers of all preschool children with smartphone addiction. The study period started from October 2019 to November 2020 in kindergarten houses of 8 districts of Tehran. Meanwhile, 150 mothers of children with smartphone addiction were selected using the purposeful sampling method. Accordingly, children who received the minimum score on smartphone addiction questionnaires were included in this study. Children with physical and mental disabilities were excluded. The inclusion criteria for the mothers were being employed, unemployed, married, and being within the age range of 20-45. The exclusion criteria were mothers heading the household. The Cochran's formula was used to determine the sample size. Considering the statistical population of the kindergartens and the possibility of the mothers to participate in the study, the sample size was 150. 
Accordingly, children and their mothers were selected using the purposeful sampling method. The sampling method was purposive because only mothers whose children received a smartphone overuse score above 75 participated in this study, according to the cellphone over-use scale [20]. Based on the method of this study, both questionnaires were distributed simultaneously. After completing the questionnaires, they were collected and analyzed by SPSS V23.

The participants were briefed on the purpose of the research, the group funding it, the way the findings would be used, if there would be any potential adverse impacts of their participation, and those having access to the findings. Written informed consent was obtained from the participants who could make an informed decision on if they wanted to participate in the evaluation. Besides, the study was approved by the Ethics Committee of Azad University of Tehran under Ethics Code IR.IAU.IAUT.REC.1399.054.

The following statistical methods were used for data analysis in this study. Besides, descriptive statistical methods, such as frequency, percentage, mean, and deviation were calculated. In addition, the Pearson's correlation coefficient and the independent t-test were used to compare the variables. For this purpose, the Cellphone Overuse Scale (COS), the Beck Depression Questionnaire, and the Intellectual Rumination Questionnaire were utilized.

Cellphone Overuse Scale (COS): Following the problematic increase in the cellphone use, Genaro et al developed a questionnaire to measure its frequency of use [20]. The cellphone overuse scale is a 23-item questionnaire designed to measure traumatic cellphone use. This scale is based on 10 psychological indicators from the Diagnostic and Statistical Manual of Mental Disorders (DSM). The cellphone overuse scale (COS) has no subscales and is scored on a six-point Likert scale of 1 to 6 (never, almost never, often, almost always, always). The Persian version of this scale has 21 questions. Each question is scored from 1 to 6 , and the 21 questions indicate the total score of the subject. The higher a person's score is, the more it will indicate his or her excessive use of cellphones. Once one achieves their grades, they will fall into one of the following classes of overuse, common users, and rare users. In the class of overuse, one gets a score above 75 on the questionnaire. In the class of common users, one gets a score of 26 to 75 on the questionnaire. In the class of rare users, one scores 25 and lower on the questionnaire. In a study on evaluating validity and reliability of the Persian version of this questionnaire, 21 items were identified as valid. Besides, the validity of the test was calculated by Cronbach's alpha at 0.90 [21].

Beck Depression Inventory - Second Edition (IIBDI): This is a revised form of the Beck Depression Inventory developed to measure severity of depression [22]. This tool has 21 items scored on a four-point Likert scale (strongly disagree: 0 , disagree: 1 , agree: 2 , and strongly disagree: 3 ), with the scores ranging from 0 to 63 . Scores of this tool are measured by a combination of the items' scores, with a higher score indicating more depression. Each of the 21 items corresponding to a symptom of depression is summed up to give a single score for the Beck Depression Inventory-II (BDI-II). There is a fourpoint scale for each item ranging from 0 to 3 . Cutoff score guidelines for the BDI-II are given upon the recommendation that thresholds be adjusted based on the characteristics of the sample in line with the purpose for the use of the BDI-II. Accordingly, the total score of $0-13$ is considered within the minimal range, $14-19$ is within the mild range, $20-28$ is within the moderate range, and 29-63 is within the severe range. Beck and Clark reported reliability of this inventory using Cronbach's alpha at 0.89 [23]. Besides, its internal consistency was 0.94 , which was estimated by the Cronbach's alpha method [24]. In the present study, the reliability coefficient of Cronbach's alpha was 0.91 .

Intellectual Rumination Questionnaire: Having been developed by Nolen Hoxma and Murrow, this questionnaire assesses four different types of negative mood reactions [25]. The Response Styles Questionnaire consists of two ruminative response scales and a distractor response scale. Besides, each item is scored on a four-point Likert scale ranging from 1 (never) to 4 (always). The total score ranges from 22 to 88 , with higher scores indicating higher degrees of ruminative symptoms. Bagherinejad et al [26] reported the validity of this questionnaire to have been between 0.88 to 0.92 , and the reliability of the retest was 0.67 . The correlation between the scores of this questionnaire and the scores of depression and anxiety in a sample of Iranian students was 0.63. In addition, the Cronbach's alpha in this study was 0.88 .

\section{Results}

The total number of the sample of mothers of children (from two to seven years old) was 150, with $55 \%$ of whom being $21-30$ years old, $33 \%$ being $31-40$ years old, and $1.7 \%$ being over 40 years old. Besides, $20 \%$ of them were employed, and $80 \%$ were unemployed. Among them, 23\% 
$(n=23)$ had a degree less than junior secondary school, $42 \%(n=42)$ had a degree over higher secondary school, and 35\% $(n=35)$ had higher education degrees.

As Table 1 shows, the mean depression of the unemployed mothers was higher than that of the employed ones. Besides, the results show that the mean rumination of the unemployed mothers was higher than that of the employed mothers.

Table 1. Descriptive statistics of mothers in this study $(n=150)$

\begin{tabular}{cccccc}
\hline Variables & Group & Mean & $\mathbf{\pm S D}$ & Min & Max \\
\hline \multirow{2}{*}{ Depression } & Employed & 3.83 & 3.78 & 0 & 9 \\
\cline { 2 - 6 } & Unemployed & 8.48 & 7.83 & 1 & 32 \\
\hline \multirow{2}{*}{ Rumination } & Employed & 14.42 & 8.34 & 6 & 49 \\
\cline { 2 - 6 } & Unemployed & 24.8 & 13.65 & 7 & 38 \\
\hline
\end{tabular}

As Table 2 shows, the coefficient of correlation between depression and rumination in mothers $(r=$ $0.604, p=0.001$ ) was significant at $p<0.05$. The results showed that the relationship between depression and rumination in mothers was significantly positive. In other words, an increase in the score of depression was correlated with an increase in the score of rumination in the mothers.

Table 2. Pearson's coefficient of correlation between depression and rumination among mothers $(n=150)$

\begin{tabular}{cccc}
\hline Variable predictor & \multicolumn{3}{c}{ Rumination } \\
\hline \multirow{2}{*}{ Depression } & $\mathrm{R}$ & Sig. \\
\cline { 2 - 4 } & & 0.604 & $\mathrm{p}=0.001$ \\
\hline
\end{tabular}

As Table 3 shows, the difference between the mean depression of the employed mothers and that of the unemployed ones $(t=1.99, P=0.04)$ was significant at $p<0.04$. According to the results, the mean depression of the unemployed mothers was higher than that of the employed mothers.

Table 3 shows the difference in the scores of the variable of rumination between the employed and unemployed mothers in the independent t-test. As Table 3 shows, the difference in the mean rumination between the employed and unemployed mothers ( $\mathrm{t}=2.34, \mathrm{P}=0.02$ ) was significant. Accordingly, the results show that the mean rumination of the unemployed mothers was higher than that of the employed ones.

Table 3. The independent t-test for comparing variables of mothers in this study ( $n=150)$

\begin{tabular}{|c|c|c|c|c|c|c|c|}
\hline Variables & Groups & Mean & SD & Df. & Mean differences & $\mathbf{T}$ & Sig. \\
\hline \multirow[b]{2}{*}{ Depression } & Employed & 3.83 & 3.78 & \multirow[b]{2}{*}{148} & \multirow{2}{*}{4.64} & \multirow{2}{*}{1.99} & \multirow{2}{*}{0.04} \\
\hline & Unemployed & 8.48 & 7.83 & & & & \\
\hline \multirow{2}{*}{ Rumination } & Employed & 14.42 & 8.34 & \multirow{2}{*}{148} & \multirow{2}{*}{9.66} & \multirow{2}{*}{2.34} & \multirow{2}{*}{0.02} \\
\hline & Unemployed & 24.08 & 13.65 & & & & \\
\hline
\end{tabular}

Table 4 shows coefficients of the correlation between rumination and depression among mothers with children addicted to smartphones. Based on the obtained results, the calculated correlation coefficients were significantly positive at the alpha level of 0.01 ( $p<0.01)$. The positive coefficients obtained indicate a direct relationship between the variables.

Table 4. Pearson's coefficient of the correlation between rumination and depression among mothers with children addicted to smartphones $(n=150)$

\begin{tabular}{cccccc}
\hline & $\mathbf{M}$ & SD & Rumination & Depression & $\begin{array}{c}\text { Smartphone } \\
\text { addiction }\end{array}$ \\
\hline Rumination & 19.24 & 10.43 & 1 & & \\
\hline Depression & 6.31 & 5.6 & $.604^{* *}$ & 1 & 1 \\
\hline Smartphone addiction & 93.62 & 14.11 & $.561^{* *}$ & $.586^{* *}$ & 1 \\
\hline
\end{tabular}

${ }^{*}$ Correlation is significant at the 0.05 level.

${ }^{* *}$ Correlation is significant at the 0.01 level 
Table 5 shows the results of regression analysis for predicting smartphone addiction through rumination and depression. According to the table, the multiple correlation coefficient between the independent variables and the dependent variables was 0.639 . Besides, the value of the coefficient of determination (R-squares) was equal to 0.401 , which shows that rumination and depression explained $40.1 \%$ of the variance in smartphone addiction. Given the number of the tstatistics obtained, being significant at the alpha level of 0.01 , the research hypothesis is confirmed. Accordingly, it is concluded that rumination and depression are significantly able to predict smartphone addiction. Besides, rumination ( $P$ $<0.01, \beta=0.324)$ and depression $(P<0.01, \beta=$ 387 ) can be used to predict smartphone addiction among children.

Table 5. Linear regression analysis for children with smartphone addiction in terms of rumination and depression among mothers $(n=150)$

\begin{tabular}{ccccccccc}
\hline Predictor variables & $\boldsymbol{B}$ & $\boldsymbol{S E}$ & $\boldsymbol{T}$ & $\boldsymbol{p}$ & $\boldsymbol{R}$ & ${\text { Adjusted } \mathbf{R}^{2}}^{2}$ & $\boldsymbol{F}$ & $\boldsymbol{p}$ \\
\hline Rumination & .324 & .249 & 4.035 & .001 & \multirow{2}{*}{.639} & .401 & \multirow{2}{*}{50.78} & .001 \\
\hline Depression & .387 & .304 & 4.819 & .001 & & & & \\
\hline
\end{tabular}

\section{Discussion}

The present study aimed to determine the relationship between depression and rumination in mothers of preschool children with smartphone addiction, and to compare depression and rumination in employed and unemployed mothers. According to the results, there is a significant positive relationship between depression and rumination in mothers of 2- to 7-year-old children. In other words, an increase in the depression score is associated with an increase in the score of rumination in mothers of children aged 2 to 7 . This result is in line with many studies with different samples, such as studies of Michl et al [15], Karabati et al [16], Sun et al [17], Hasegawa et al [18], and Hasegawa et al [19]. Hasegawa et al reported that although rumination predicted the extent of subsequent depression, depression predicted higher levels of rumination at subsequent points of time [18]. In the same vein, many longitudinal studies have shown that depressive symptoms are effective and positive predictors of subsequent rumination [27-28].

In an experience sampling study that explored the correlation between the state of ruminative selffocus and negative effects, a reciprocal relationship was found between rumination and depression [25]. However, these processes are known to be aspects of depressive rumination [29]. Moreover, the results show that the mean depression and rumination in the unemployed mothers were higher than those in the employed ones. Therefore, the authors of this study try to rationally explain this finding. According to our direct question, given the results of the study, both groups faced mental problems (rumination and depression) due to their inability to avoid the stressful use of cellphones by their children. Besides, the employed mothers suffered from mental problems, such as depression and rumination, less than the unemployed ones [3033].

Some studies have explained their results more accurately. Accordingly, employed mothers consider work, kids, household, and duties as the most common stressors, while unemployed ones consider kids and finance as stressors. Employed mothers often switch between home and work. Finally, one can infer that the wellbeing of working and non-working mothers is significantly different. Accordingly, employed mothers deal with various situations both at home and in their workplace [32]. Although there are many studies indicating an association between unemployment and mental health impairments, the mediating processes and mechanisms by which unemployment influences mental health are not well known [33]. A recent study suggests that financial strain, frustration of life goals, social support, and dysfunctional health behaviors, such as depression and rumination, may mediate negative effects of unemployment on health [33].

In addition, the unemployed mothers shared a lack of motivation and dedication as the reasons for their failure to cope with their children's problems. However, given the behavioral problems of certain children, there are many mediating factors, such as financial problems, which directly affect rumination and depression in unemployed mothers. Therefore, unemployment and financial issues can be defined as the significant factors affecting mental health in unemployed mothers. Findings from several studies and meta-analyses indicated negative effects of unemployment on psychological well-being. Furthermore, epidemiological studies found the higher prevalence of common mental disorders, such as severe depression and rumination, in the unemployed people [32]. 
A study examined differences between quantitative, mental, and emotional household demands, as well as stress and depression among employed and non-employed women. According to the results, despite the presence of no differences in the stress level between working and nonworking women, depression was more in working women than in non-working ones [33].

Thus, these findings were inconsistent with the present results. The prominent strength of this study was its being the only study to have used an observational measure of maternal depression and rumination with smartphone addiction in their children. A significant limitation of this study was its being correlational and comparable in nature, so it was not possible to infer causality. Therefore, it cannot be concluded that low maternal positivity causes the development of smartphone addiction in children. Accordingly, the results could not be applicable to more socially and ethnically diverse samples. Future research is recommended to be conducted to compare employed and unemployed mothers in causing smartphone addiction in children.

In this study, the sampling method was used at random, so the results are not generalizable. In future research, it is suggested that random sampling be done. To be more confident of the results achieved, researchers need to address this issue more broadly in the future. In addition, given the results, it is recommended that in future research, mediating variables, such as the number of children, economic conditions, parental conflicts, and other factors, be examined.

\section{Conclusion}

In summary, the present study demonstrated a significant association between depression and rumination in unemployed mothers of children with smart phone addiction. Moreover, the results showed that the mean depression in unemployed mothers was higher than that in employed ones. Besides, the mean rumination of the unemployed mothers was higher than that of the employed mothers. The findings provide a basis for doing follow-up research on designing a novel and effective prevention program to prevent depression and rumination in mothers of children with smartphone addiction.

\section{Acknowledgement}

The authors would like to extend their gratitude to all mothers and children whose participation made this study possible.
Conflict of interest: None declared.

\section{References}

1. Thimbleby $\mathrm{H}$. Technology and the future of healthcare. J Public Health Res 2013; 2(3):e28.

2. Parasuraman S, Sam AT, Yee SWK, Chuon BLC, Ren LY. Smartphone usage and increased risk of mobile phone addiction: A concurrent study. Int J Pharm Investig 2017; 7(3):125-31.

3. Kwon M, Kim DJ, Cho H, Yang S. The smartphone addiction scale: development and validation of a short version for adolescents. PLoS One 2013; 8(12):e83558.

4. Alavi AH, Buttlar WG. An Overview of Smartphone Technology for Citizen-Centered, Real-Time and Scalable Civil Infrastructure Monitoring. Future Gener Comput Syst 2019; 93(2019):651-72.

5. Kuss DJ, Kanjo E, Crook-Rumsey M, Kibowski F, Wang GY, Sumich A. Problematic Mobile Phone Use and Addiction Across Generations: The Roles of Psychopathological Symptoms and Smartphone Use. J Technol Behav Sci 2018; 3(3):141-9.

6. Cheever NA, Rosen LD, Carrier LM, Chavez A. Out of sight is not out of mind: The impact of restricting wireless mobile device use on anxiety levels among low, moderate and high users. Comput Human Behav 2014; 37:290-7.

7. Panova T, Lleras A. Avoidance or boredom: Negative mental health outcomes associated with use of Information and Communication Technologies depend on users' motivations. Comput Human Behav 2016; 58:249-58.

8. Cha SS, Seo BK. Smartphone use and smartphone addiction in middle school students in Korea: Prevalence, social networking service, and game use. Health Psychol Open 2018; 5(1):2055102918755046.

9. Kim HH, Chun J. Is the relationship between parental abuse and mobile phone dependency (MPD) contingent across neighborhood characteristics? A multilevel analysis of Korean Children and Youth Panel Survey. PLoS One 2018; 13(5):e0196824.

10. Samaha M, Hawi NS. Relationships among smartphone addiction, stress, academic performance, and satisfaction with life. Comput Human Behav 2016; 57:321-5.

11. Kim HJ, Min JY, Kim HJ, Min KB. Accident risk associated with smartphone addiction: A study on university students in Korea. J Behav Addict 2017; 6(4):699-707.

12. Ihm J. Social implications of children's smartphone addiction: The role of support networks and social engagement. J Behav Addict 2018; 7(2):473-81.

13. Lyubomirsky S, Layous K, Chancellor J, Nelson SK. Thinking about rumination: the scholarly contributions and intellectual legacy of Susan 
Nolen-Hoeksema. Annu Rev Clin Psychol 2015; 11:1-22.

14. Bonifacci P, Tobia V, Marra V, Desideri L, Baiocco R, Ottaviani C. Rumination and Emotional Profile in Children with Specific Learning Disorders and Their Parents. Int $\mathrm{J}$ Environ Res Public Health 2020; 17(2):389.

15. Michl LC, McLaughlin KA, Shepherd K, NolenHoeksema S. Rumination as a mechanism linking stressful life events to symptoms of depression and anxiety: Longitudinal evidence in early adolescents and adults. J Abnorm Psychol 2013; 122(2):339-52.

16. Karabati S, Ensari N, Fiorentino D. Job Satisfaction, Rumination, and Subjective WellBeing: A Moderated Mediational Model. J Happiness Stud 2019; 20(1):251-68.

17. Sun $H$, Tan $Q$, Fan G, Tsui Q. Different effects of rumination on depression: key role of hope. Int J Ment Health Syst 2014; 8:53.

18. Hasegawa A, Kunisato $Y$, Morimoto $H$, Nishimura $\mathrm{H}$, Matsuda $\mathrm{Y}$. How do Rumination and Social Problem Solving Intensify Depression? A Longitudinal Study. J Ration Emot Cogn Behav Ther 2018; 36(1):28-46.

19. Hasegawa A, Kunisato $Y$, Morimoto $H$, Nishimura $H$, Matsuda $Y$. Depressive rumination and urgency have mutually enhancing relationships but both predict unique variance in future depression: A longitudinal study. Cogent Psychology 2018; 5(1):1450919.

20. Jenaro C, Flores N, Gómez-Vela M, GonzálezGil F, Caballo C. Problematic internet and cellphone use: Psychological, behavioral, and health correlates. Addict Res Theory 2007; 15(3):309-20.

21. Golmohammadian $M, \quad$ Yaseminejad $P$. Normalization, Validity and Reliability of CellPhone Over-Use Scale (COS) among University Students. Journal of Social Psychology 2011; 6(19):37-52

22. Beck AT, Epstein N, Brown G, Steer RA. An inventory for measuring clinical anxiety: psychometric properties. J Consult Clin Psychol 1988; 56(6):893-7.

23. Beck AT, Clarck DA. Anxiety and depression: an information processing perspective. Anxiety
Res 1988; 1(1):23-36

24. Zemestani M, Davoodi I, Mehrabi-Zadeh, Honarmand M, Zargar Y. Effectiveness of Group Behavioral Activation on Depression, Anxiety and Rumination in Patients with Depression and Anxiety. Journal of Clinical Psychology 2014; 5(4):73-84 .

25. Nolen-Hoeksema S. Responses to depression and their effects on the duration of depressive episodes. J Abnorm Psychol 1991; 100(4):56982.

26. Bagherinezhad M, Salehi Fadardi J, Tabatabayi SM. The relationship between rumination and depression in a sample of Iranian student. Research in Clinical Psychology and Counseling 2010; 11(1):21-38.

27. Hasegawa A, Yoshida T, Hattori $Y$, Nishimura $\mathrm{H}$, Morimoto $\mathrm{H}$, Tanno $\mathrm{Y}$. Depressive Rumination and Social Problem Solving in Japanese University Students. J Cogn Psychother 2015; 29(2):134-52.

28. Moberly NJ, Watkins ER. Ruminative self-focus and negative affect: An experience sampling study. J Abnorm Psychol 2008; 117(2):314-23.

29. Nolen-Hoeksema S, Larson J, Grayson C. Explaining the gender difference in depressive symptoms. J Pers Soc Psychol 1999; 77(5):1061-72.

30. Sharma K, Mishra S. Comparative study of wellbeing of working and non-working women. Indian Journal of Health \& Well-being 2018; 9(5):799-801.

31. Neubert M, Süssenbach $P$, Rief W, Euteneuer $F$. Unemployment and mental health in the German population: the role of subjective social status. Psychol Res Behav Manag 2019; 12:557-64.

32. McMunn A, Kelly Y, Cable N, Bartley M. 082 Maternal employment and child socio-emotional behaviour: longitudinal evidence from the Millennium Cohort Study. J Epidemiol Community Health 2010; 64(Suppl 1):A32-3.

33. Rashid T, Mustafa S. To Measure the Level of Depression among Working and Non-Working Married Women. Annals of Punjab Medical College 2015; 9(2):95-9. 